\title{
An In Vitro Analysis of Disintegration Times of Different Formulations of Olanzapine Orodispersible Tablet: A Preliminary Report
}

\author{
David Hobbs $\cdot$ Jamie Karagianis • \\ Tamas Treuer $\cdot$ Joel Raskin
}

Published online: 30 October 2013

(C) The Author(s) 2013. This article is published with open access at Springerlink.com

\begin{abstract}
Background Orodispersible tablets (ODTs) are tablet or wafer forms of medication that disintegrate in the mouth, aided only by saliva. ODTs rely on different fast dissolve/ disintegration manufacturing technologies.

Objectives Disintegration time differences for several olanzapine ODT forms were investigated. Risperdal $\mathrm{M}-\mathrm{Tab}^{\circledR}$ was included as a non-olanzapine ODT comparator.

Research Design and Methods Eleven olanzapine ODT examples and orodispersible risperidone strengths were evaluated in vitro for formulation composition, manufacturing method, disintegration and dissolution characteristics,
\end{abstract}

This work was conducted when JK was an employee of Eli Lilly.

Electronic supplementary material The online version of this article (doi:10.1007/s40268-013-0030-8) contains supplementary material, which is available to authorized users.

D. Hobbs $(\bowtie)$

Eli Lilly and Company, Lilly Corporate Center, Indianapolis, IN 46285, USA

e-mail: hobbsdg@1illy.com

J. Karagianis

Waypoint Centre for Mental Health, 500 Church St,

Penetanguishene, ON L9M 1G3, Canada

J. Karagianis

Department of Psychiatry, University of Toronto, Toronto,

Canada

T. Treuer

Eli Lilly and Company, Madach I. u. 13-14, Budapest 1075, Hungary

J. Raskin

Eli Lilly Canada Inc., 3650 Danforth Avenue, Toronto, ON M1N

2E8, Canada and formulation differences in comparison with freeze dried Zydis ${ }^{\circledR}$ ODT. Automated dissolution test equipment captured ODT dissolution rates by measuring real-time release of active ingredient. A high-speed video camera was used to capture tablet disintegration times in warm simulated saliva. Main Outcome Measure The main outcome measure was the disintegration and dissolution characteristics of the ODT formulations.

Results The ODT manufacturing method was associated with time to disintegrate; the fastest were freeze dried tablets, followed by soft compressed tablets and then hard/dense tablets. Olanzapine Zydis ${ }^{\circledR}$ was the only ODT that completely disintegrated in less than $4 \mathrm{~s}$ for all strengths $(5,10$, 15 , and $20 \mathrm{mg}$ ), followed by $5-\mathrm{mg}$ Prolanz FAST ${ }^{\circledR}(12 \mathrm{~s})$ and then risperidone ODT $4 \mathrm{mg}$ (40 s). Reasons for slow dissolution of the olanzapine generics may include low product potency, excipient binding, excipient solubility, active ingredient particle size and incomplete disintegration.

Conclusions Differences in the formulation and manufacturing process of olanzapine ODTs appear to have a strong influence on the disintegration time of the active compound; differences that may potentially impact their use in clinical practice.

\section{Introduction}

The treatment of mental disorders usually requires prolonged pharmacotherapy in order to resolve the current episode and reduce the risk for recurrence of symptoms, while addressing the challenges of low compliance in the long term. Such prolonged therapy requires considerable commitment on the part of patients to take their medication as prescribed. Medication compliance is often challenging 
among psychiatric patients, including those with schizophrenia or bipolar disorder; this can be associated with poor long-term outcomes and, ultimately, treatment failure [1].

A greater understanding of patients' preferences for new formulations of treatment is central to current models of shared patient-doctor decision making, and has gained considerable interest in scientific research for orodispersible formulations of antidepressants and antipsychotics [2]. The effectiveness of the antipsychotic drug olanzapine classic oral tablet in the treatment of patients with schizophrenia has been widely investigated in several randomized, controlled trials, and observational studies [3$7]$ and in several meta-analyses [8,9].

In recent years, more clinical attention has been paid to oral dispersible tablet formulation of medications [10]. Lyophilized (freeze dried), orally disintegrating olanzapine is a rapid dissolving formulation of olanzapine that disintegrates in saliva almost instantaneously. The formulation was developed as a convenient, easy to ingest and potentially adherence-enhancing alternative to the standard olanzapine coated tablet. Pharmacokinetic studies have shown that the olanzapine orodispersible tablet (ODT) is bioequivalent to olanzapine standard tablet with the same rate and extent of bioavailability [11]. Clinical studies have shown that olanzapine ODTs and standard olanzapine tablets have similar efficacy and tolerability profiles; however, olanzapine ODTs appear to have a number of advantages over olanzapine standard tablets in terms of adherence, patient preference and reduction in nursing burden $[2,12,13]$.

Olanzapine ODTs may be useful for patients who have difficulty swallowing standard tablets; those with poor insight who may try to cheek or spit out their medication; those who need to have their ingestion verified but who do not want an injection; or those who prefer this formulation for other reasons. There are many generic olanzapine orodispersible formulations, but their relative disintegration and dispersion times have never been studied to our knowledge. Variation in dispersion times might be expected, depending on the different fast dissolve/disintegration technologies used to manufacture the tablets and/or the disintegration test used to evaluate them. Olanzapine $\mathrm{Zy}$ $\operatorname{dis}^{\circledR}$ (also known as Velotab ${ }^{\circledR}$ ) is manufactured by Catalent Pharma Solutions (Somerset, NJ, USA), and is made by a freeze drying process that provides a low-density, highly porous structure that readily disintegrates in the oral cavity. Although bioequivalence is accepted for generic ODTs, the time it takes for an ODT to disintegrate and dissolve in the oral cavity may potentially impact clinical parameters such as patient acceptance and adherence to treatment.

For olanzapine Zydis ${ }^{\circledR}$ ODT, the elapsed time for initial and complete disintegration was measured in two small in vivo studies $[14,15]$. However, these studies used different methods: one took the first measurement of initial disintegration at $15 \mathrm{~s}$, while the other took the first measurement at $5 \mathrm{~s}$. It is desirable to compare disintegration times among different products using the same methodology. Given the obvious challenges of standardizing in vivo assessments, the objective of our current in vitro comparison was to investigate in vitro disintegration time and dissolution rate differences of various generic formulations of olanzapine ODT relative to olanzapine Zydis ${ }^{\circledR}$ in simulated saliva. We also compared the chemical and physical properties of each ODT and measured in vitro disintegration time for risperidone ODT [16] as a comparator.

\section{Materials and Methods}

All types of olanzapine ODT that could be obtained were evaluated (Table 1). Eleven different examples were filmed to determine disintegration times, and all were evaluated for manufacturing method, dissolution characteristics and formulation differences, including the freeze dried Zydis ${ }^{\circledR}$ formulation of olanzapine ODT and risperidone ODT. A Canon XHL1 HD camera (Canon, Tokyo, Japan) was used to capture a 3-min disintegration event for each ODT product added to $30 \mathrm{~mL}$ of non-agitated $37^{\circ} \mathrm{C}$ (initial temperature) simulated saliva solution in a $10-\mathrm{cm}$ Petri dish. Disintegration was defined as the time it took a tablet to reach full dispersion after addition to the artificial saliva (see Table 2 for the formulation, based on formulations described in Giannola et al. [17] and Gal et al. [18]). Drug product excipient data were obtained from published product literature. Dose form and manufacturing method (compressed tablet, lyophilized wafer) were determined by microscopic/visual observation.

Dissolution testing used a USP Apparatus \#2, DISTEK DISBA0045 and DISBA0046 with an Opt-Diss UV fiber optic SPEC0088 attachment (Distek Inc., North Brunswick, NJ, USA). The system quantified the solubilized antipsychotic in $500 \mathrm{~mL}$ of $37^{\circ} \mathrm{C}$ simulated saliva every $10 \mathrm{~s}$ for $6 \mathrm{~min}$, and then every minute for $14 \mathrm{~min}$, with paddle speeds of 20 or $30 \mathrm{rpm}$ to simulate the oral cavity environment [16] (Table 3). Agitation was then increased $150 \mathrm{rpm}$ for an additional $16 \mathrm{~min}$ to release all available olanzapine. Olanzapine active ingredient standard was used to calibrate the system, and dissolution was repeated a minimum of three times. The Distek dissolution apparatus was calibrated with three standards for each of the 12 probes (two dissolution baths with six vessels each) and a standard absorbance curve was calculated for each probe. If the relative standard deviation was too high, the probe was not used. Care was taken to randomize the analysis within the vessels available and thus provide assurance of 
Table 1 Drug names and manufacturing information

\begin{tabular}{|c|c|c|c|c|c|c|c|}
\hline $\begin{array}{l}\text { Commercial } \\
\text { name }\end{array}$ & Manufacturer & $\begin{array}{l}\text { Strength } \\
(\mathrm{mg})\end{array}$ & $\begin{array}{l}\text { Manufacturing } \\
\text { method }\end{array}$ & Expiration & $\begin{array}{l}\text { Lot } \\
\text { number }\end{array}$ & $\begin{array}{l}\text { Country } \\
\text { of origin }\end{array}$ & Distributor \\
\hline Risperdal M-Tab ${ }^{\circledR}$ & $\begin{array}{l}\text { Janssen Pharmaceuticals } \\
\text { Inc. }\end{array}$ & 2 & $\begin{array}{l}\text { Freeze dried } \\
\text { tablet }\end{array}$ & 06/2012 & 0JG018 & USA & $\begin{array}{l}\text { Janssen Pharmaceuticals } \\
\text { Inc. }\end{array}$ \\
\hline Risperdal M-Tab $^{\circledR}$ & $\begin{array}{l}\text { Janssen Pharmaceuticals } \\
\text { Inc. }\end{array}$ & 4 & $\begin{array}{l}\text { Freeze dried } \\
\text { tablet }\end{array}$ & $01 / 2012$ & 0BG1274 & USA & $\begin{array}{l}\text { Janssen Pharmaceuticals } \\
\text { Inc. }\end{array}$ \\
\hline $\begin{array}{l}\text { Novo-Olanzapine } \\
\text { OD }^{\circledR}\end{array}$ & Teva Pharmaceutical & 5 & Molded tablet & $01 / 2013$ & 03400081 & Canada & Nova Pharm \\
\hline Olanzapine $\mathrm{FT}^{\circledR}$ & ABL Pharma & 5 & $\begin{array}{l}\text { Compressed } \\
\text { tablet }\end{array}$ & $02 / 2012$ & B0683A & Chile & ABL Pharma Peru SAC \\
\hline Olanzapine ODT $^{\circledR}$ & Sandoz Canada Inc. & 5 & $\begin{array}{l}\text { Compressed } \\
\text { tablet }\end{array}$ & 03/2012 & 0000876 & Canada & Sandoz Canada Inc. \\
\hline Olaxinn $^{\circledR}$ & $\begin{array}{l}\text { Ali Raif Ilac San. A.s. } \\
\text { (ARIS) }\end{array}$ & 5 & $\begin{array}{l}\text { Compressed } \\
\text { tablet }\end{array}$ & $04 / 2012$ & 10040845 & Turkey & $\begin{array}{l}\text { Generica Ilac San.ve } \\
\text { Tic. }\end{array}$ \\
\hline $\begin{array}{l}\text { pms-Olanzapine }_{\text {ODT }^{\circledR}}\end{array}$ & PharmaScience Inc. & 5 & $\begin{array}{l}\text { Compressed } \\
\text { tablet }\end{array}$ & $07 / 2011$ & C000303 & Canada & PharmaScience Inc. \\
\hline Prolanz FAST $^{\circledR}$ & $\begin{array}{r}\text { Procaps S.A., } \\
\text { Barranquilla }\end{array}$ & 5 & $\begin{array}{l}\text { Compressed } \\
\text { tablet }\end{array}$ & $06 / 2012$ & 0062447 & Columbia & NA \\
\hline Zolrix $^{\circledR}$ & $\begin{array}{l}\text { KRKA Polska Sp., } \\
\text { Varsava }\end{array}$ & 5 & $\begin{array}{l}\text { Compressed } \\
\text { tablet }\end{array}$ & $01 / 2012$ & $\begin{array}{l}\text { P14110- } \\
0110\end{array}$ & Poland & Salus, Ljubljana, d.d. \\
\hline Zyprexa $^{\circledR}$ Zydis $^{\circledR}$ & Eli Lilly and Company & 5 & $\begin{array}{l}\text { Freeze dried } \\
\text { wafer }\end{array}$ & $06 / 2013$ & 1076944 & Britain & Eli Lilly and Company \\
\hline Anzapine ORO ${ }^{\circledR}$ & Okasa Pharma Pvt. Ltd & 10 & $\begin{array}{l}\text { Compressed } \\
\text { tablet }\end{array}$ & 08/2010 & S88053 & India & Laboratoire BIO VITAL \\
\hline Lanzaprex $^{\circledR}$ & $\begin{array}{l}\text { El Kendi Industrie du } \\
\text { Med. }\end{array}$ & 10 & $\begin{array}{l}\text { Compressed } \\
\text { tablet }\end{array}$ & 09/2012 & $\mathrm{L} 10 \mathrm{C} 2$ & Algeria & NA \\
\hline Olanzapine $\mathrm{FT}^{\circledR}$ & ABL Pharma & 10 & $\begin{array}{l}\text { Compressed } \\
\text { tablet }\end{array}$ & $02 / 2012$ & B0735A & Chile & ABL Pharma Peru SAC \\
\hline Prolanz FAST $^{\circledR}$ & $\begin{array}{l}\text { Procaps S.A., } \\
\text { Barranquilla }\end{array}$ & 10 & $\begin{array}{l}\text { Compressed } \\
\text { tablet }\end{array}$ & $04 / 2012$ & 0041462 & Columbia & NA \\
\hline Tanssel D ${ }^{\circledR}$ & Okasa Pharma Pvt. Ltd & 10 & $\begin{array}{l}\text { Compressed } \\
\text { tablet }\end{array}$ & 06/2011 & SJ9016 & India & $\begin{array}{l}\text { Biocross S.A. } \\
\text { Guatemala }\end{array}$ \\
\hline Zyprexa $^{\circledR}$ Zydis $^{\circledR}$ & Eli Lilly and Company & 10 & $\begin{array}{l}\text { Freeze dried } \\
\text { tablet }\end{array}$ & $06 / 2013$ & 1076944 & Britain & Eli Lilly and Company \\
\hline $\begin{array}{l}\text { CO Olanzapine } \\
\text { ODT }^{\circledR}\end{array}$ & Cobalt Pharmaceuticals & 15 & $\begin{array}{l}\text { Compressed } \\
\text { tablet }\end{array}$ & $06 / 2012$ & BX411 & Canada & Cobalt Pharmaceuticals \\
\hline $\begin{array}{l}\text { pms-Olanzapine }_{\text {ODT }^{\circledR}} \\
\end{array}$ & PharmaScience Inc. & 15 & $\begin{array}{l}\text { Compressed } \\
\text { tablet }\end{array}$ & $07 / 2011$ & $\mathrm{C} 000305$ & Canada & PharmaScience Inc. \\
\hline Zyprexa $^{(}{ }^{\circledR}$ Zydis $^{\circledR}$ & Eli Lilly and Company & 15 & $\begin{array}{l}\text { Freeze dried } \\
\text { tablet }\end{array}$ & $04 / 2013$ & 1058967 & Britain & Eli Lilly and Company \\
\hline $\begin{array}{l}\text { Novo-Olanzapine } \\
\text { OD }^{\circledR}\end{array}$ & Teva Pharmaceutical & 20 & Molded tablet & $11 / 2012$ & 93440011 & Canada & Nova Pharm \\
\hline Olaxinn $^{\circledR}$ & $\begin{array}{l}\text { Ali Raif Ilac San. A.s. } \\
\text { (ARIS) }\end{array}$ & 20 & $\begin{array}{l}\text { Compressed } \\
\text { tablet }\end{array}$ & $04 / 2012$ & 10040848 & Turkey & $\begin{array}{l}\text { Generica Ilac San.ve } \\
\text { Tic. }\end{array}$ \\
\hline Olanzapine ODT $^{\circledR}$ & Sandoz Canada Inc. & 20 & $\begin{array}{l}\text { Compressed } \\
\text { tablet }\end{array}$ & $12 / 2011$ & 0000012 & Canada & Sandoz Canada Inc. \\
\hline Zolrix $^{\circledR}$ & $\begin{array}{l}\text { KRKA Polska Sp., } \\
\text { Varsava }\end{array}$ & 20 & $\begin{array}{l}\text { Compressed } \\
\text { tablet }\end{array}$ & $10 / 2011$ & $\begin{array}{l}\text { P14065- } \\
1009\end{array}$ & Poland & Salus, Ljubljana, d.d. \\
\hline Zyprexa $^{\circledR}$ Zydis $^{\circledR}$ & Eli Lilly and Company & 20 & $\begin{array}{l}\text { Freeze dried } \\
\text { tablet }\end{array}$ & $04 / 2013$ & 1067672 & Britain & Eli Lilly and Company \\
\hline
\end{tabular}

$O D T$ orodispersible tablet

$N A$ not available 
Table 2 Simulated saliva formulation

\begin{tabular}{ll}
\hline Ingredient & $\begin{array}{l}\text { Grams/liter of } \\
\text { purified water }\end{array}$ \\
\hline Sodium chloride $(\mathrm{NaCl})$ & 0.126 \\
Potassium chloride $(\mathrm{KCl})$ & 0.964 \\
Potassium thiocyanide $(\mathrm{KSCN})$ & 0.189 \\
Potassium phosphate monobasic $\left(\mathrm{KH}_{2} \mathrm{PO}_{4}\right)$ & 0.655 \\
Urea & 0.200 \\
Sodium sulfate $\left(\mathrm{Na}_{2} \mathrm{SO}_{4} 10 \mathrm{H}_{2} \mathrm{O}\right)$ & 0.763 \\
Ammonium chloride $\left(\mathrm{NH}_{4} \mathrm{Cl}\right)$ & 0.178 \\
Calcium chloride dihydrate $\left(\mathrm{CaCl}_{2} 2 \mathrm{H}_{2} \mathrm{O}\right)$ & 0.228 \\
Sodium bicarbonate $\left(\mathrm{NaHCO}_{3}\right)$ & 0.631 \\
\hline
\end{tabular}

Table 3 Orodispersible tablet dissolution conditions [19]

\begin{tabular}{|c|c|}
\hline Parameter & Equipment/Measure \\
\hline $\begin{array}{r}\text { Dissolution } \\
\text { apparatus }\end{array}$ & DISBA0045, DISBA0046 (Distek 6100) \\
\hline Configuration & Paddles (USP apparatus 2) \\
\hline Temperature & $37^{\circ} \mathrm{C}$ \\
\hline Medium & Simulated saliva \\
\hline Volume & $500 \mathrm{~mL}$ \\
\hline $\begin{array}{l}\text { Rotational } \\
\text { speed }\end{array}$ & $30 \mathrm{rpm}$ \\
\hline Analysis & $\begin{array}{l}\text { SPEC0088 (Distek Opt-Diss Fiber Optic UV } \\
\text { dissolution system) }\end{array}$ \\
\hline Wavelength & $\begin{array}{l}255 \mathrm{~nm} \text { (with blank subtraction at } 330 \mathrm{~nm} \text { ) for } \\
\text { olanzapine } \\
276 \mathrm{~nm} \text { (with blank subtraction at } 330 \mathrm{~nm} \text { ) for } \\
\text { risperidone }\end{array}$ \\
\hline $\begin{array}{l}\text { Frequency of } \\
\text { readings }\end{array}$ & $\begin{array}{l}\text { Every } 10 \mathrm{~s} \text { from } 0 \text { to } 6 \mathrm{~min} \\
\text { Every } 1 \mathrm{~min} \text { from } 6 \text { to } 20 \mathrm{~min} \\
\text { Then change paddle speed to at least } 150 \mathrm{rpm} \text { and } \\
\text { take one reading at } 30 \mathrm{~min} \text { and at } 90 \mathrm{~min}\end{array}$ \\
\hline
\end{tabular}

comparable results of tests performed in triplicate on each generic tablet. Initial disintegration was quick and difficult to differentiate among some products, so the time to first measurable concentration of active ingredient in the dissolution media (simulated saliva) was used as a proxy, since the onset of dissolution is normally preceded by disintegration.

\section{Results}

\subsection{Disintegration Times (Time Taken to Reach Full Dispersion)}

We found that the method of ODT manufacture (see Table 1 for manufacturing details for all compounds tested) had the greatest influence on the time for disintegration; in general, the fastest were freeze dried tablets, then soft compressed tablets and then hard/dense tablets. Olanzapine Zydis ${ }^{\circledR}$ was the only ODT that completely disintegrated in less than $4 \mathrm{~s}$ for all strengths $(5,10,15$, and $20 \mathrm{mg}$; Table 4). The second fastest disintegration time was Prolanz FAST ${ }^{\circledR}(5 / 10 \mathrm{mg} ; 12 \mathrm{~s})$, followed by risperidone (4 mg; $40 \mathrm{~s}$ ).

In the agitated aqueous media of the dissolution vessel (rather than the sedentary environment of the Petri dish just described), both olanzapine Zydis ${ }^{\circledR}$ and risperidone ODT disintegrated immediately upon coming into contact with aqueous media; however, the active compound (olanzapine) in the Zydis ${ }^{\circledR}$ tablets dissolved faster in the simulated saliva media ( $\mathrm{pH}$ 6.8) than the risperidone in the risperidone ODTs. Surprisingly, risperidone 2-mg ODT disintegrated slower than the 4-mg with double the mass, and was potentially influenced by the shape and density of the tablet. Other products varied in their disintegration characteristics, but essentially remained as a clump that did not always fully disperse when physically agitated after $3 \mathrm{~min}$ of standing without mixing. Compressed tablets consistently had a higher amount of visible residue at the end of the 3-min evaluation period.

\subsubsection{Dissolution Times (Release of Active Product)}

Using time to dissolution as a proxy for disintegration, several generics required $20 \mathrm{~s}$ or more to initiate release of the drug substance (Table 5) and required both increasing the agitation rate, and additional time $(\sim 30 \mathrm{~min})$ to maximize dissolution. In this evaluation, only four of the drug products tested released more than $80 \%$ of the active ingredient within the first $10 \mathrm{~min}$. Release for all but Zolrix ${ }^{\circledR}$ was around $90 \%$ or above after applying $150 \mathrm{rpm}$ for $10 \mathrm{~min}$ at the end of the analysis.

\subsubsection{5-mg Olanzapine and 4-mg Risperidone ODTs}

Figures 1 and 2 are a summary of the 5-mg data at 30-rpm paddle speed for the first $3 \mathrm{~min}$ and first $30 \mathrm{~min}$, respectively. When examining the first $3 \mathrm{~min}$ (Fig. 1) of the dissolution profile, the olanzapine Zydis ${ }^{\circledR}$ formulation is the first to release active compound, with dissolution over $30 \%$ in less than $60 \mathrm{~s}$, twice as fast as the 4-mg risperidone ODT. The Prolanz FAST ${ }^{\circledR}$ 5-mg formulation is also rapid and after $1 \mathrm{~min}$ had higher, although more variable, release (Fig. 1). Three samples (olanzapine Zydis ${ }^{\circledR}$, Prolanz FAST $^{\circledR}$, and Novo-Olanzapine $\mathrm{OD}^{\circledR}$ ) were run again at the lower agitation speed to explore potential differences between the products; at $20 \mathrm{rpm}$, only olanzapine Zydis ${ }^{\circledR}$ disintegrated instantly, and Prolanz FAST ${ }^{\circledR}$ had a noticeable delay in the low-agitation environment. Novo-Olanzapine $\mathrm{OD}^{\circledR}$, a molded tablet, also had a faster dissolution profile than the remainder of the samples (Fig. 1). 
Table 4 Tablet excipients and disintegration

\begin{tabular}{|c|c|c|}
\hline $\begin{array}{l}\text { Commercial name and } \\
\text { dose }\end{array}$ & Excipients & Disintegration observations \\
\hline $\begin{array}{l}\text { ABL Olanzapine } \mathrm{FT}^{\circledR} \\
10 \mathrm{mg} \text {; Zapinex } \mathrm{FT}^{\circledR} \\
5,10 \mathrm{mg}\end{array}$ & $\begin{array}{l}\text { Lactose monohydrate, hydroxypropyl cellulose, crospovidone, } \\
\text { magnesium stearate, glycerin diacetate, colloidal silicon } \\
\text { dioxide, microcrystalline cellulose, polyethylene glycol } 8000 \text {, } \\
\text { mint powder }\end{array}$ & $\begin{array}{l}\text { Yellow and red, compressed tablet, stops swelling } \\
\text { in media after 55-75 s, disintegration }>180 \mathrm{~s}^{\mathrm{a}}\end{array}$ \\
\hline Anzapine $\mathrm{ORO}^{\circledR} 10 \mathrm{mg}$ & $\begin{array}{l}\text { Lactose monohydrate, hydroxypropyl cellulose, sodium } \\
\text { cyclamate, magnesium stearate, talc }\end{array}$ & $\begin{array}{l}\text { Compressed tablet, stops swelling in media after } \\
85 \mathrm{~s} \text {, coarse disintegration }>180 \mathrm{~s}^{\mathrm{a}}\end{array}$ \\
\hline $\begin{array}{l}\text { CO Olanzapine ODT } \\
15 \mathrm{mg}\end{array}$ & Not available & $\begin{array}{l}\text { Pale yellow, round, compressed tablet, } \\
\text { disintegration }>180 \mathrm{~s}^{\mathrm{a}}\end{array}$ \\
\hline Lanzaprex $^{\circledR} 10 \mathrm{mg}$ & $\begin{array}{l}\text { Lactose, crospovidone, hydroxypropyl cellulose, aerosil, talc, } \\
\text { magnesium stearate, mint aroma }\end{array}$ & $\begin{array}{l}\text { Compressed tablet, stops swelling in media after } \\
44 \mathrm{~s} \text {, disintegration complete at }<180 \mathrm{~s}^{\mathrm{b}}\end{array}$ \\
\hline $\begin{array}{l}\text { Novo-Olanzapine } \mathrm{OD}^{\circledR} \\
5,15,20 \mathrm{mg}\end{array}$ & $\begin{array}{l}\text { Mannitol, sodium starch glycolate, dextrose, flavoring, } \\
\text { (tartrazine), FD\&C yellow \#5 aluminum lake }\end{array}$ & $\begin{array}{l}\text { Yellow (5), pink (15), and blue (20), round, } \\
\text { compression molded tablet fully wetted in } 5 \mathrm{~s} \text {, } \\
\text { disintegrated in }<180 \mathrm{~s}^{\mathrm{b}}\end{array}$ \\
\hline Olaxinn ${ }^{\circledR} 5,20 \mathrm{mg}$ & $\begin{array}{l}\text { Crospovidone, lactose monohydrate, colloidal silicon dioxide, } \\
\text { hydroxypropyl cellulose, talc, magnesium stearate, mint } \\
\text { powder }\end{array}$ & $\begin{array}{l}\text { Yellow, round, compressed tablet fully wetted in } \\
5-9 \mathrm{~s} \text {, disintegrated in }<180 \mathrm{~s}^{\mathrm{b}}\end{array}$ \\
\hline $\begin{array}{l}\text { pms-Olanzapine } \text { ODT }^{\circledR} \\
15,5 \mathrm{mg}\end{array}$ & $\begin{array}{l}\text { Aspartame, colloidal silicon dioxide, crospovidone, guar gum, } \\
\text { magnesium stearate, mannitol, microcrystalline cellulose, } \\
\text { pregelatinized starch, sodium lauryl sulfate }\end{array}$ & $\begin{array}{l}\text { Yellow, round, compressed tablet fully wetted in } \\
5 \mathrm{~s} \text {, disintegrated in }<180 \mathrm{~s}^{\mathrm{b}}\end{array}$ \\
\hline $\begin{array}{l}\text { Prolanz FAST }{ }^{\circledR} 5 \\
\quad 10 \mathrm{mg}\end{array}$ & $\begin{array}{l}\text { Lactose monohydrate, hyprolose, crospovidone, } \\
\text { microcrystalline cellulose, magnesium stearate }\end{array}$ & $\begin{array}{l}\text { Red, } 5 / 10-\mathrm{mg} \text {, compressed tablet, disintegrated in } \\
7-12 \mathrm{~s} \text {, coarse dispersion at } 180 \mathrm{~s}\end{array}$ \\
\hline $\begin{array}{l}\text { Sandoz Olanzapine } \\
\text { ODT }^{\circledR} 20 \mathrm{mg}\end{array}$ & $\begin{array}{l}\text { Colloidal silicon dioxide, crospovidone, flavoring, mint } \\
\text { powder, hydroxypropyl cellulose, lactose monohydrate, } \\
\text { magnesium stearate, talc }\end{array}$ & $\begin{array}{l}\text { Compressed tablet, stops swelling in media after } \\
85 \mathrm{~s} \text {, disintegrated at } 180 \mathrm{~s}\end{array}$ \\
\hline Zolrix $^{\circledR} 20 \mathrm{mg}$ & $\begin{array}{l}\text { Mannitol, microcrystalline cellulose, crospovidone, } \\
\text { hydroxypropyl cellulose, aspartame, calcium silicate, } \\
\text { magnesium stearate }\end{array}$ & $\begin{array}{l}\text { Compressed tablet, disintegrated in media after } \\
25 \mathrm{~s} \text {, coarse dispersion at } 180 \mathrm{~s}\end{array}$ \\
\hline $\begin{array}{l}\text { Zyprexa }^{\circledR} \text { Zydis }^{\circledR} 5,10, \\
15,20 \mathrm{mg}\end{array}$ & Mannitol, aspartame, gelatin, preservatives & Fully dispersed in $4 \mathrm{~s}$ \\
\hline $\begin{array}{l}\text { Risperdal M-Tab }{ }^{\circledR} 2 \text {, } \\
\quad 4 \mathrm{mg}\end{array}$ & $\begin{array}{l}\text { Colloidal silicone dioxide, hypromellose, lactose, magnesium } \\
\text { stearate, microcrystalline cellulose, propylene glycol, sodium } \\
\text { lauryl sulfate, corn starch, colorant }\end{array}$ & $\begin{array}{l}\text { Red, 4-mg, round wafer, fully wetted at } 3 \mathrm{~s} \text {, } \\
\text { disintegrated in } 40 \mathrm{~s}, 2-\mathrm{mg} \text { pillow is slower and } \\
\text { lumps }\end{array}$ \\
\hline
\end{tabular}

$O D T$ orodispersible tablet

a Disintegration was not complete at $180 \mathrm{~s}$. When the Petri dish was stirred, there was clearly core material still intact

b Disintegration was a slow erosion of the core tablet, and it was not obvious when disintegration was complete; yet when stirred at the end of $180 \mathrm{~s}$, there was no evidence of tablet core material (lump). In these cases, the tablet was said to have disintegrated in $<180 \mathrm{~s}$, but significantly more slowly than $\mathrm{Zydis}^{\circledR}$ or risperidone (minutes instead of seconds)

As shown in Fig. 1, olanzapine Zydis ${ }^{\circledR}$ is the first to initiate disintegration and shows a steady rate of dissolution, whereas some of the generic ODTs were well below $100 \%$ even at the 30-min dissolution time point (Fig. 2).

\subsubsection{0-mg Tablets}

The Prolanz FAST ${ }^{\circledR}$ formulation has a quick dissolution time, but shows a longer delay to catch up to the Zydis ${ }^{\circledR}$ formulation, taking $2 \mathrm{~min}$ before they are equivalent (data not shown; see Figs. 1, 2 for 5-mg dose profiles). At a lower agitation rate of $20 \mathrm{rpm}$, olanzapine Zydis ${ }^{\circledR} 10 \mathrm{mg}$ still has the fastest dissolution rate in the first $3 \mathrm{~min}$, and olanzapine Zydis ${ }^{\circledR}$ dissolution is not significantly affected by dosage strengths $(5,10 \mathrm{mg})$. However, the Prolanz
FAST $^{\circledR}$ dissolution rate is affected by the increased mass of the tablet.

\subsubsection{5-mg Tablets}

At $20 \mathrm{~min}$, the generic ODTs released less than $60 \%$ of active compound, while olanzapine Zydis ${ }^{\circledR}$ released $95 \%$. At the 90-min time point, and with increased agitation, the generic ODTs reached $96-112 \%$ release.

\subsubsection{0-mg Tablets}

The olanzapine Zydis ${ }^{\circledR}$ ODT formulation is the fastest to disintegrate and dissolve. With a longer dissolution time (90 $\mathrm{min}$ ) and increased agitation, all products were close to 
Table 5 Time to first measurable concentration at $30 \mathrm{rpm}$

\begin{tabular}{|c|c|c|c|c|}
\hline \multirow[t]{2}{*}{ Product name } & \multicolumn{4}{|c|}{ Time, percentage released (s, \%) by formulation strength } \\
\hline & $5 \mathrm{mg}$ & $10 \mathrm{mg}$ & $15 \mathrm{mg}$ & $20 \mathrm{mg}$ \\
\hline ABL Olanzapine $\mathrm{FT}^{\circledR}$ & 0,1 & $10,1^{\mathrm{a}} 40$ & - & - \\
\hline Anzapine $\mathrm{ORO}^{\circledR}$ & - & 30,1 & - & - \\
\hline ARIS Olaxinn $^{\circledR}$ & $0,1^{\mathrm{a}} 5$ & - & - & 20,1 \\
\hline CO Olanzapine ODT ${ }^{\circledR}$ & - & - & $20,2^{\mathrm{a}} 25$ & - \\
\hline Lanzaprex $^{\circledR}$ & - & $15,1^{\mathrm{a}} 35$ & - & - \\
\hline Novo-Olanzapine OD ${ }^{\circledR}$ & $10,3^{\mathrm{a}} 15$ & - & - & 30,1 \\
\hline pms-Olanzapine ODT ${ }^{\circledR}$ & $10,2^{a} 15$ & - & 20,1 & - \\
\hline Prolanz FAST $^{\circledR}$ & 20,8 & $10,2^{a} 25$ & - & - \\
\hline Sandoz Olanzapine ODT ${ }^{\circledR}$ & $20,1^{\mathrm{a}} 25$ & - & - & $20,1^{\mathrm{a}} 25$ \\
\hline Tanssel D ${ }^{\circledR}$ & - & $30,1^{a} 35$ & - & - \\
\hline Zolrix $^{\circledR}$ & $0,1^{\mathrm{a}} 5$ & - & - & $20,1^{\mathrm{a}} 25$ \\
\hline Zydis $^{\circledR}$ & $0,4^{\mathrm{a}} 5$ & 5,7 & $0,1^{\mathrm{a}} 5$ & 0,1 \\
\hline Risperdal M-Tab ${ }^{\circledR b}$ & 10,2 & - & - & - \\
\hline
\end{tabular}

$O D T$ orodispersible tablet

${ }^{a}$ Unadjusted time as per graph. Some graphs did not start at zero

${ }^{\mathrm{b}}$ Data shown are for the 4-mg dose

Fig. 1 Summary of 5-mg dissolution data at $30 \mathrm{rpm}$, up to $3 \mathrm{~min}$. ODT orodispersible tablet

Fig. 2 Summary of 5-mg dissolution data at $30 \mathrm{rpm}$, up to $30 \mathrm{~min}$. $O D T$ orodispersible tablet
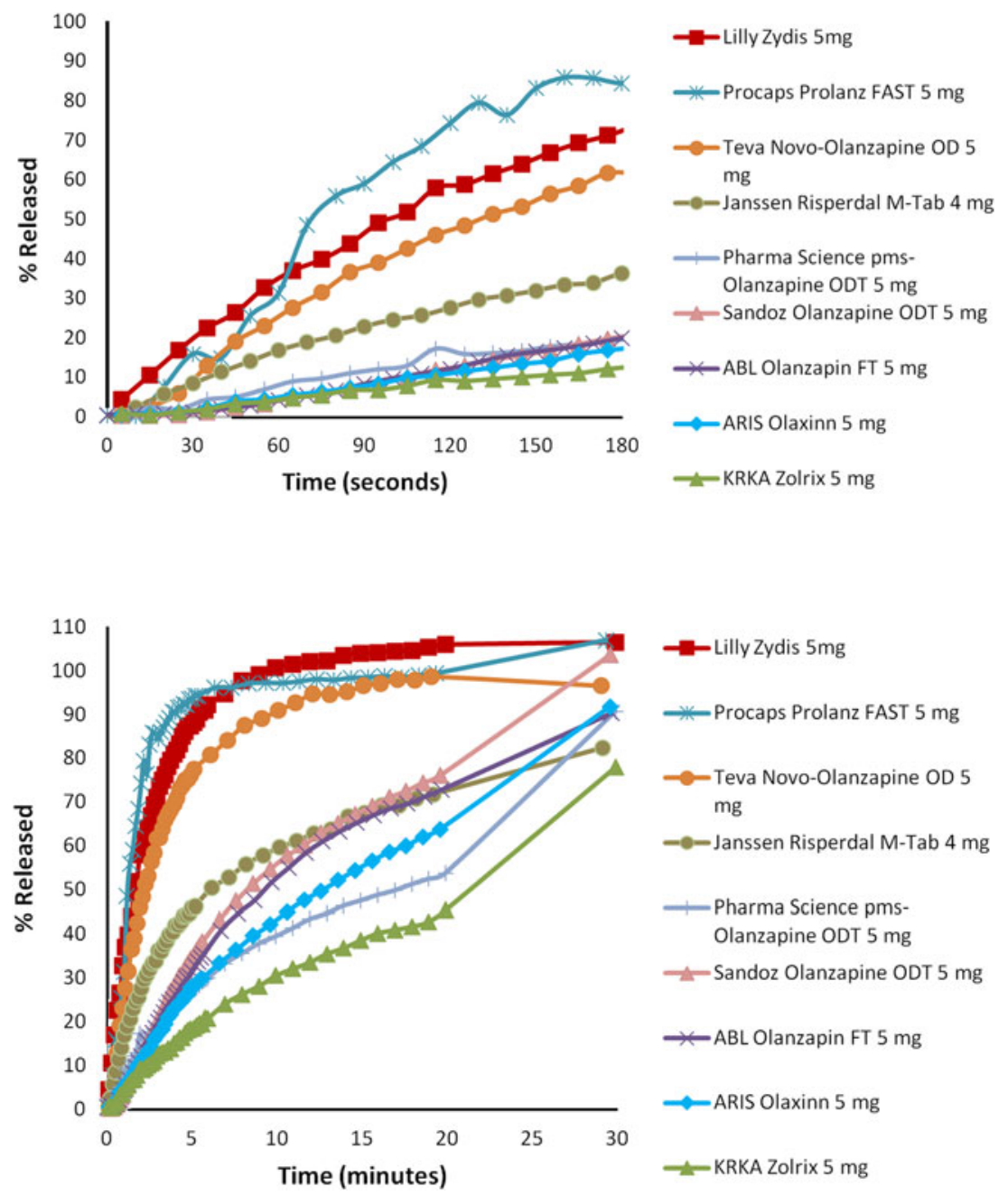
$100 \%$ released at the final time point. The freeze dried ODT dissolution profiles are very similar regardless of the tablet mass or active ingredient content. Generic ODT formulations using conventional compression or molding methods of manufacture were significantly slower to dissolve as the mass of the tablet increased.

\section{Discussion}

Based on our results, we found potentially important differences between ODT formulations manufactured with different technologies. The simulated saliva in vitro dissolution test may be considered a proxy for the disintegration process in a patient's mouth because it mimics the oral cavity environment and solutions. Differences in ODT formulation, manufacturing process, and tablet mass are associated with different disintegration times, which may have a potential impact on their use in clinical practice. Different disintegration times and tablet residue could influence mouth feel and the ability to swallow unaided by fluids, which could, in turn, influence adherence to treatment.

It is important to note that several generic tablet disintegration rates are slow enough to permit 'cheeking' and expectoration of the medication. Surreptitious rejection of medication by patients occurs sometimes in clinical practice [15]. If a tablet is swallowed and the $\mathrm{pH}$ becomes more acidic, the olanzapine will dissolve more rapidly than in the more neutral $\mathrm{pH}$ of saliva; however, the time for complete disintegration may be no better than in the mouth. Clinicians need to be aware of the potential differences among products, because it could differentially influence the success of this behavior. The use of polymeric excipients, which swell in water to speed disintegration, may inhibit rapid and complete dissolution of the active ingredient in some formulations.

There are three possible explanations for the low final dissolution values $(<90 \%)$ after $30 \mathrm{~min}$ in the dissolution bath: (1) olanzapine is not very soluble in aqueous solutions close to $\mathrm{pH} \mathrm{7,} \mathrm{and} \mathrm{in} \mathrm{these} \mathrm{formulations,} \mathrm{active}$ pharmaceutical ingredient particle size may make a difference; (2) the drug is trapped or bound to excipients; or (3) analytical interference from the excipients. At $20 \mathrm{~min}$, generics released less than $60 \%$, while olanzapine Zydis ${ }^{\circledR}$ released $95 \%$. With the longer time point $(90 \mathrm{~min}$ ), they reached 96-112\% release. Generic ODT formulations using loosely compressed tablets had relatively fast and/or coarse disintegration but slow dissolution. Olanzapine Zydis $^{\circledR}$ (a freeze dried tablet) was the fastest disintegrating ODT formulation and exhibited the most effective dissolution curve of all the tablet strengths tested, regardless of potency. The investigated generic olanzapine ODT products required more than $30 \mathrm{~s}$ to dissolve even $10 \%$ of the active ingredient when compared with olanzapine $\mathrm{Zy}$ dis ${ }^{\circledR}$ ODT, which could release approximately $25 \%$ in the same time period.

Generic ODT products use different manufacturing platforms: direct compression; molded tablets; uncoated tablets; and some with pigment colorants. Risperdal M-Tab ${ }^{\circledR}$ and olanzapine Zydis ${ }^{\circledR}$ tablets may have similar disintegration rates, but the Zydis ${ }^{\circledR}$ ODT dissolved at twice the speed (likely due to the differences in active ingredient solubility in artificial saliva). In our tests, the smaller mass of the 5-mg olanzapine ODTs may facilitate the observed shorter disintegration and dissolution times versus the larger 20-mg tablets. Generic olanzapine ODT formulations incorporate water expansive polymers that appeared in the dispersion as a coarse insoluble residue, which may explain slow dissolution rates. After $5 \mathrm{~min}$, some generic forms of olanzapine ODT almost match the dissolution rate of $\mathrm{Zy}$ dis ${ }^{\circledR}$ but do not realize $100 \%$ release.

There are some limitations of our experiments. The in vitro disintegration times may not be identical to in vivo disintegration times, and the small number of generic drug tablets available to the investigation did not permit statistical analysis.

\section{Conclusions}

The in vitro artificial saliva disintegration and dissolution tests are a proxy for the disintegration process in a patient's mouth. Tablet orodispersibles are consistently slower to disintegrate and release drug substance than lyophilized wafers. Compared with olanzapine Zydis ${ }^{\circledR}$ ODT, generic olanzapine ODT formulations of soft compressed tablets incorporate water expansive polymers that appeared in the dispersion as a coarse insoluble residue, which may explain their slow dissolution rates. Furthermore, in a direct comparison between risperidone ODT and olanzapine Zydis ${ }^{\circledR}$, orodispersible drugs with similar manufacturing methods (lyophilization), it is evident that, even though disintegration rates are similar, the risperidone is not as soluble in artificial saliva as is olanzapine. By using these test methods, differences in the formulation and manufacturing process of ODTs were seen to have a strong influence on the disintegration and dissolution of the drug products; differences that may potentially affect their use in clinical practice.

Acknowledgments All authors meet the International Committee of Medical Journal Editors (ICJME) authorship criteria, and no one qualifying for authorship has been excluded. This research was funded by Eli Lilly and Company, Indianapolis, Indiana, USA. The authors would also like to gratefully acknowledge Stacy Osborne for analytical support. The results were originally presented in a poster 
format at the WFSBP Congress 2011, Prague, 29 May-2 June 2011 [20].

Author contributions All authors were involved in the development and writing of this manuscript, and all approved the final version.

Conflict of interest David Hobbs, Tamas Treuer, and Joel Raskin are employees of Eli Lilly and Company, the manufacturer of olanzapine. Jamie Karagianis is a former employee of Eli Lilly and Company. Lilly laboratories conducted the main tests, and all authors participated in the design of the experiment and in the interpretation of the results.

Open Access This article is distributed under the terms of the Creative Commons Attribution Noncommercial License which permits any noncommercial use, distribution, and reproduction in any medium, provided the original author(s) and the source are credited.

\section{References}

1. Mohamed S, Rosenheck R, McEvoy J, et al. Cross-sectional and longitudinal relationships between insight and attitudes toward medication and clinical outcomes in chronic schizophrenia. Schizophr Bull. 2009;35(2):336-46.

2. Bitter I, Treuer T, Dilbaz N, et al. Patients' preference for olanzapine orodispersible tablet compared with conventional oral tablet in a multinational, randomized, crossover study. World J Biol Psychiatry. 2010;11(7):894-903.

3. Kahn RS, Fleischhacker WW, Boter H, et al. Effectiveness of antipsychotic drugs in first-episode schizophrenia and schizophreniform disorder: an open randomised clinical trial. Lancet. 2008;371:1085-97.

4. Lieberman JA, Stroup TS, McEvoy JP, et al. Effectiveness of antipsychotic drugs in patients with chronic schizophrenia. N Engl J Med. 2005;353:1209-23.

5. Novick D, Haro JM, Suarez D, et al. Symptomatic remission in previously untreated patients with schizophrenia: 2-year results from the SOHO study. Psychopharmacology. 2007;191:1015-22.

6. Bitter I, Treuer T, Dyachkova Y, et al. Antipsychotic prescription patterns in outpatient settings: 24-month results from the Intercontinental Schizophrenia Outpatient Health Outcomes (ICSOHO) study. Eur Neuropsychopharmacol. 2008;18:170-80.

7. Dossenbach M, Pecenak J, Szulc A, et al. Long-term antipsychotic monotherapy for schizophrenia: disease burden and comparative outcomes for patients treated with olanzapine, quetiapine, risperidone, or haloperidol monotherapy in a pan-continental observational study. J Clin Psychiatry. 2008;69:1901-15.

8. Leucht S, Komossa K, Rummel-Kluge C, et al. A meta-analysis of head-to-head comparisons of second-generation antipsychotics in the treatment of schizophrenia. Am J Psychiatry. 2009;166(2): 152-63.

9. Leucht S, Corves C, Arbter D, et al. Second-generation versus first-generation antipsychotic drugs for schizophrenia: a metaanalysis. Lancet. 2009;373(9657):31-41.

10. Ghosh T, Ghosh A, Prasad D. A review on new generation orodispersible tablets and its future prospective. Int J Pharm Pharm Sci. 2011;1:1-7.

11. Bergstrom RF, Mitchell M, Witcher J, et al. Rapid onset of absorption with olanzapine orally disintegrating tablets. J Emerg Nurs. 2004;30(5):416-7.

12. San L, Casillas M, Ciudad A, et al. Olanzapine orally disintegrating tablet: a review of efficacy and compliance. Review. CNS Neurosci Ther. 2008;14(3):203-14.

13. Karagianis J, Grossman L, Landry J, et al. A randomized controlled trial of the effect of sublingual orally disintegrating olanzapine versus oral olanzapine on body mass index: the PLATYPUS Study. Schizophr Res. 2009;113:41-8.

14. Chue P, Jones B, Taylor CC, et al. Dissolution profile, tolerability, and acceptability of the orally disintegrating olanzapine tablet in patients with schizophrenia. Can J Psychiatry. 2002;47(8): $771-4$.

15. Chue P, Welch R, Binder C. Acceptability and disintegration rates of orally disintegrating risperidone tablets in patients with schizophrenia or schizoaffective disorder. Can J Psychiatry. 2004;49(10):701-3.

16. Daily Med. Risperdal M-Tab (risperidone), orally disintegrating tablets: Summary of product characteristics [online]. http:// dailymed.nlm.nih.gov/dailymed/lookup.cfm?setid=7e117c7e-02fc4343-92a1-230061dfc5e0. Accessed 5 December 2012.

17. Giannola LI, De Caro V, Giandalia G, Siragusa MG, Tripodo C, Florena AM, Campisi G. Release of naltrexone on buccal mucosa: permeation studies, histological aspects and matrix system design. Eur J Pharm Biopharm. 2007;67:425-33.

18. Gal JY, Fovet Y, Adib-Yadzi. About a synthetic saliva for in vitro studies. Talanta. 2001;53:1103-15.

19. Chue P, Prinzo RS, Binder CE. Do formulation switches exacerbate existing medical illness? Results of an open-label transition to orally disintegrating risperidone tablets. Hum Psychopharmacol. 2007;22(5):307-14.

20. Hobbs D, Karagianis J, Treuer T, et al. An in vitro analysis of disintegration times of different formulations of orally disintegrating olanzapine [abstract plus poster]. 10th World Congress of Biological Psychiatry; 2011 May 29-Jun 02; Prague. 